\title{
Engraftment Syndrome
}

National Cancer Institute

\section{Source}

National Cancer Institute. Engraftment Syndrome. NCI Thesaurus. Code C63324.

A toxicity of hematopoietic stem cell transplantation that manifests as fever, rash and pulmonary deterioration which becomes evident at marrow engraftment. It occurs unexpectedly and is occasionally fatal. It can occur after an autogeneic or an allogeneic hematopoietic cell transplantation. 